\title{
Wave Breaking in Dense Plumes
}

\author{
PAUl R. HOLLAND \\ British Antarctic Survey, Cambridge, United Kingdom \\ RICHARD E. HEWITT \\ School of Mathematics, University of Manchester, Manchester, United Kingdom \\ MATTHEW M. SCASE \\ School of Mathematical Sciences, University of Nottingham, Nottingham, United Kingdom
}

(Manuscript received 9 May 2013, in final form 5 November 2013)

\begin{abstract}
Sinking dense plumes are important in many oceanographic settings, notably the polar formation of deep and bottom waters. The dense water sources feeding such plumes are commonly affected by tidal modulation, leading to plume variability on short time scales. In a simple unsteady theory of one-dimensional plumes (based on conservation equations for volume, momentum, and buoyancy), this plume variability is manifested as waves that travel down the resulting current. Using numerical techniques applied to the hyperbolic conservation equations, this study investigates the novel concept that these waves may break as they travel down the plumes, triggering intense local mixing between the dense fluid and surrounding ocean. The results demonstrate that the waves break at geophysically relevant distances from the plume source. The location of wave breaking is very sensitive to plume drag from the seabed, the properties of the dense source, and the amplitude and period of the source modulation. To the extent that the simple model represents the real world, these results suggest that wave breaking originating from the tidal modulation of dense plumes could lead to a strong and previously unexplored source of local deep-ocean mixing.
\end{abstract}

\section{Introduction}

The global oceans are filled with dense deep and bottom waters formed at the poles. Around Antarctica, dense shelf waters, formed by sea ice growth over the continental shelf seas, cascade down the continental slopes into the deep ocean, mixing with ambient waters to form the Antarctic Bottom Water that underlies the majority of the world's oceans (Baines and Condie 1998; Orsi et al. 1999). Similar cascades in the Arctic create a pool of denser water that contributes to the dense waters overflowing the Greenland-Scotland Ridge, which in turn feed the North Atlantic Deep Water that occupies much of the Atlantic Ocean (Hansen and Østerhus 2000; Ivanov et al. 2004).

Corresponding author address: Paul Holland, British Antarctic Survey, High Cross, Madingley Road, Cambridge CB3 0ET, United Kingdom.

E-mail: p.holland@bas.ac.uk
A common feature of these cascades and overflows is that they occur in relatively shallow shelf-edge regions where tidal amplitudes are large (Padman and Erofeeva 2004; Padman et al. 2009). This implies that a full understanding of the formation of globally important dense water masses requires knowledge of the effects of shortperiod modulation of dense water sinking down seabed slopes. Other dense plumes are also affected by pulsation at the source, such as the tidally affected Mediterranean outflow into the North Atlantic (Nash et al. 2012), Atlantic overflow into the deep Caribbean (MacCready et al. 1999), and the diurnally controlled sinking of dense nearshore waters in lakes and seas (Fer et al. 2001; Biton et al. 2008).

The most intensively studied tidally affected plume is in the Ross Sea, Antarctica, which is dominated by strong diurnal tides with a significant spring-neap cycle (Whitworth and Orsi 2006; Muench et al. 2009; Padman et al. 2009; Wang et al. 2010). The effect of tides can be broken down into three major categories: oscillating 
advection of the plume path, temporal variation of the shear-driven mixing experienced by the plume, and pulsation of the plume source. Using a simple model of unsteady one-dimensional plumes, Holland (2011) investigated the effects of source pulsation and variable shear, finding that the impact of pulsation in the plume source gave best agreement with the observations.

This raises an important possibility. A pulsed source produces waves that travel down the plume, and simple analytical investigation of a reduced system suggests that these waves should break within $50 \mathrm{~km}$ of the source (Holland 2011). If such wave breaking does occur on geophysically relevant scales, then it will lead to rapid, localized mixing between dense water and its ambient fluid, with important consequences for the depth and characteristics of the deep water masses produced. However, previous studies have been unable to conclusively demonstrate the presence of wave breaking, as a result of the inability of their numerical schemes to represent a discontinuous solution and the possibility that numerical diffusion prevents modeled waves from breaking.

The processes governing mixing between oceanic plumes and their environment are summarized by Cenedese and Adduce (2010), and references therein. There are three primary sources of mixing: shear at the plume-ambient interface, shear or roughness effects in the bottom boundary layer, and hydraulic jumps. Interfacial instability is usually held to be dominant in ocean plumes, but superimposing a barotropic tidal forcing on the plume increases the importance of boundary-driven mixing (Muench et al. 2009). Internal hydraulic jumps will lead to vigorous and localized mixing (Holland et al. 2002), but their frequency and distribution in the oceans are basically unknown. If the breaking of waves shed from a temporally modulated dense source can occur on geophysically relevant scales, it would add an important additional source of mixing between dense plumes and their environment, with a local and vigorous character that is similar to hydraulic jumps.

In this study, we use modern numerical techniques applied to a hyperbolic system of conservation equations for volume, momentum, and buoyancy, in order to investigate wave breaking of one-dimensional plumes emanating from a temporally modulated source. The numerical methodology is chosen to accurately represent the behavior near a discontinuity associated with the breaking wave and to minimize the effects of numerical diffusion, which may otherwise artificially prevent waves from breaking. The factors preventing previous studies from considering wave breaking are therefore alleviated. Another important consideration comes from the work of Scase and Hewitt (2012), who show that unsteady extensions of the classical axisymmetric buoyant rising plume model of Morton et al. (1956) are ill posed. Clearly, establishing the well-posedness of the conservative model of a nondiffusive line plume descending a slope is therefore a necessary prerequisite for this study.

\section{Modeling}

In an effort to simplify the effects of tidal modulation of source conditions on the evolution of dense plumes, Holland (2011) formulated an unsteady, onedimensional model subjected to unsteady ambient flow and/or modulation of the source. "Top hat" profiles were assumed for the plume quantities. The most restrictive assumption of this model is the neglect of Coriolis force, which is justified solely by a desire for simplicity that should be borne in mind when evaluating the conclusions. The effects of ambient flow were found to be less consistent with observations than those of a pulsedbuoyancy source, so only the latter is pursued here. In the absence of ambient motion, the Holland (2011) model for conservation of volume, momentum, and buoyancy requires

$$
\begin{aligned}
\frac{\partial D}{\partial t}+\frac{\partial}{\partial x}(D U) & =E \sin \theta U \\
\frac{\partial}{\partial t}(D U)+\frac{\partial}{\partial x}\left(D U^{2}\right) & =g^{\prime} D\left(\sin \theta-\frac{\partial D}{\partial x}\right)-c U^{2} \\
\frac{\partial}{\partial t}\left(D g^{\prime}\right)+\frac{\partial}{\partial x}\left(D U g^{\prime}\right) & =0
\end{aligned}
$$

where $D$ is the plume depth, $U$ is the velocity, $g^{\prime}$ is the reduced gravity, $\theta$ is the seabed slope angle, $c$ is a drag coefficient, and $E$ is an entrainment constant. Holland (2011) considered a variety of forms for the entrainment term on the right-hand side of (1a). In this study, we employ a conventional formulation in which entrainment, $E \sin \theta U$, is simply proportional to seabed slope and plume speed (Bo Pedersen 1980); however, a range of values of $E$ will be considered [including the value used by Holland (2011)] to assess the sensitivity of the results. The fluid outside the plume is stagnant, so entrainment does not add momentum, and the interfacial stress at the plume-ambient interface is neglected as much smaller than the seabed stress.

In the remainder of this paper we will use a slightly different form of (1). The derivation of (1b) makes an approximation, (9) of Holland (2011), that neglects $\left(2 \rho_{0}\right)^{-1} g^{\prime} D^{2} \partial \rho / \partial x$, where $g^{\prime}=\left(\rho-\rho_{a}\right) g / \rho_{0}$ for a reference density $\rho_{0}$ and an ambient density $\rho_{a}$. A reintroduction of this previously neglected term allows (1) to be written in a conservative form, which is beneficial for the 
numerical formulation when discontinuous solutions are to be obtained.

A change of variables from $\left(D, U\right.$, and $\left.g^{\prime}\right)$ to the plume depth, volume flux, and cross-sectional total buoyancy ( $D, Q=U D$, and $\left.B=D g^{\prime}\right)$ allows the (hyperbolic) system to be written as

$$
\begin{gathered}
S \frac{\partial D}{\partial t}+\frac{\partial Q}{\partial x}=E \sin \theta \frac{Q}{D} \\
S \frac{\partial Q}{\partial t}+\frac{\partial}{\partial x}\left(\frac{Q^{2}}{D}+\frac{1}{2} B D\right)=B \sin \theta-c\left(\frac{Q}{D}\right)^{2}, \quad \text { and } \\
S \frac{\partial B}{\partial t}+\frac{\partial}{\partial x}\left(\frac{B Q}{D}\right)=0 .
\end{gathered}
$$

Here we have nondimensionalized using a mean source plume depth $D_{s}$, mean source reduced gravity $g_{s}^{\prime}$, and mean source (gravity wave) speed $U_{s}=\sqrt{g_{s}^{\prime} D_{s}}$. The parameter $S$ then defines the relative time scales $S=$ $T^{-1} D_{s} / U_{s}$, where $T$ is the period of the modulation in plume source properties and $D_{s} / U_{s}$ is the time taken for a parcel of fluid to travel a distance of one plume thickness.

For the above nondimensionalization, a steady plume solution of (2) exists in the form

$$
D=1+\bar{D} x, \quad Q=(1+\bar{D} x) \bar{U}, \quad \text { and } \quad B=1,
$$

where $\bar{D}=E \sin \theta$ and the plume velocity is constant:

$$
\bar{U}^{2}=\sin \theta\left(\frac{1-E / 2}{E \sin \theta+c}\right) .
$$

Following the tidal-modulation approach of Holland (2011), we construct a periodically forced system by imposing the analogous unsteady source conditions:

$D(x=0, t)=D_{0}(t), \quad B(x=0, t)=B_{0}(t), \quad$ and

$Q(x=0, t)=U_{0}(t) D_{0}(t)$,

with

$$
\begin{gathered}
D_{0}(t)=1+A_{p} \sin (2 \pi t), \\
B_{0}(t)=1+A_{p} \sin (2 \pi t), \quad \text { and } \\
U_{0}(t)=\left[B_{0}(t) \sin \theta \frac{1-E / 2}{E \sin \theta+c}\right]^{1 / 2},
\end{gathered}
$$

where $A_{p}$ is an amplitude of pulsation; we recover the steady solution when $A_{p}=0$. The source velocity for $U_{0}$ above is chosen such that it remains consistent with the governing model equations in the quasi-steady limit.

Throughout this paper, we consider the results of a central baseline simulation and parameter sensitivity around it. For consistency with Holland (2011), we choose baseline values broadly appropriate to the Ross Sea, Antarctica. Specifically, we use $c=0.003$ and $\theta=0.01$, source properties of $D_{s}=100 \mathrm{~m}$ and $g_{s}^{\prime}=0.002 \mathrm{~m} \mathrm{~s}^{-2}$, and a modulation period of $T=24 \mathrm{~h}$ (Holland 2011), giving $S=0.002588$. The entrainment coefficient appearing in (1a) will be chosen to be $E=0.072$, following Bo Pedersen (1980).

\section{Model stability}

The work of Scase and Hewitt (2012) showed that the unsteady analog (Scase et al. 2006) of the axisymmetric plume model first introduced by Morton et al. (1956) is ill posed; a comparable result has also been demonstrated in the context of momentum-driven laminar boundary layers by Hewitt and Duck (2011). This ill-posedness of unsteady plume models arises from the rapid downstream growth of linear high-frequency/small-wavelength perturbations to the (otherwise well established and successful) steady solutions. The downstream growth of small-amplitude perturbations increases without bound as the frequency increases, which inevitably leads to any time-marching numerical method being fundamentally flawed. Given these new results, it is first prudent to consider the wellposedness of the comparable Holland (2011) model applied to unsteady dense plumes on shallow slopes.

For the original formulation, (1), a dimensional steady solution is given by

$D_{B}=E \sin (\theta) x, \quad U_{B}=\sin (\theta)\left[\frac{g_{s}^{\prime} x_{s} E(1-E)}{E \sin (\theta)+c}\right]^{1 / 2}, \quad$ and

$g_{B}^{\prime}=g_{s}^{\prime} \frac{x_{s}}{x}$,

where subscript $B$ denotes the steady "base" solution and subscript $s$ denotes a quantity at the source, that is, $x_{s}$ is the position of the source and $g_{s}^{\prime}$ is the reduced gravity at the source. Following Scase and Hewitt (2012), we may perturb this steady state (for $\delta \ll 1$ ) by

$D=D_{B}[1+\delta \cdot \Delta(\xi, \tau)], \quad U=U_{B}[1+\delta \cdot u(\xi, \tau)], \quad$ and $g^{\prime}=g_{B}^{\prime}[1+\delta \cdot \gamma(\xi, \tau)]$,

using the nondimensional variables defined by $\xi=$ $x \omega / U_{B}$ and $\tau=\omega t$ for some constant frequency $\omega$. Furthermore, we consider a single Fourier mode such that 

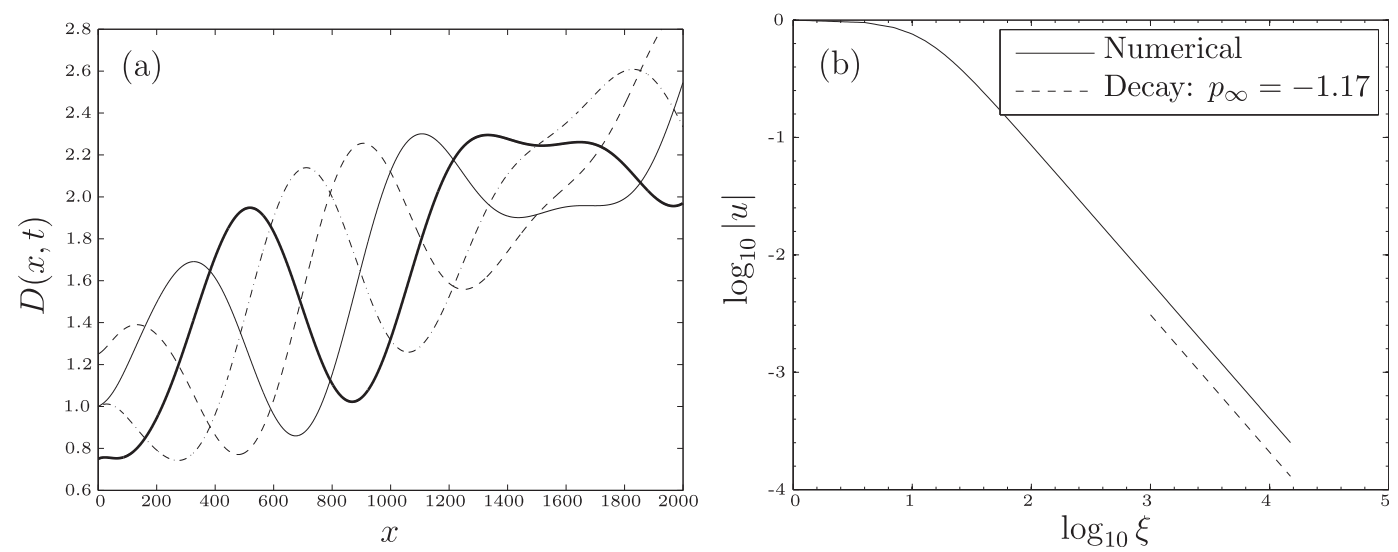

FIG. 1. Solutions to the linearized model equation [(7)] for the base case $D_{s}=100 \mathrm{~m}, g_{s}^{\prime}=0.002 \mathrm{~m} \mathrm{~s}^{-2}, \theta=0.01, T=$ $24 \mathrm{~h}, E=0.072$, and $c=0.003$. (a) The steady solution (5a) has been multiplied by the perturbation $\left(1+A_{p} \Delta\right)$, with $A_{p}=0.25$, for comparison with Fig. 3a. Time has been nondimensionalized by the period of oscillation and the line styles correspond to $\tau=0$ (dashed), $1 / 4$ (thin solid), 1/2 (thick solid), and 3/4 (dot-dashed). Quantities shown are dimensionless, where $D$ has been nondimensionalized by $D_{s}$ and $x$ by $x_{s}$. (b) The decay of the instability is in agreement with (9), with $p_{\infty}=-1.17$.

$\Delta(\xi, \tau)=\Delta(\xi) \exp \{i \tau\}, u(\xi, \tau)=u(\xi) \exp \{i \tau\}$, and $\gamma(\xi)$ $\exp \{i \tau\}$, where $i$ is the usual unit imaginary number. Thus, (1) transforms to become at $O(\delta)$

$$
\begin{aligned}
\xi \frac{d}{d \xi}(\Delta+u)= & -\Delta(1+i \xi), \\
\left(\frac{1+E \kappa}{1-E}\right) \xi \frac{d \Delta}{d \xi}+2 \xi \frac{d u}{d \xi}= & {\left[\kappa\left(\frac{1-2 E}{1-E}\right)-\frac{E}{1-E}-i \xi\right] \Delta } \\
& -[2(1+\kappa)+i \xi] u+(1+\kappa) \gamma \\
\frac{d}{d \xi}(\Delta+u+\gamma)= & -i(\Delta+\gamma),
\end{aligned}
$$

where $\kappa=c /(E \sin \theta)$.

We can rearrange to find the following second order ODE for $u$ :

$$
\begin{gathered}
\frac{d^{2} u}{d \xi^{2}}+\frac{4+3 \kappa-(5+4 \kappa) E+2 i \xi(1-E)}{[1-E(2+\kappa)] \xi} \frac{d u}{d \xi} \\
-\frac{[\xi-i(3+2 \kappa)](1-E)}{[1-E(2+\kappa)] \xi} u=0 .
\end{gathered}
$$

This equation supports solutions of the form of a sum of Whittaker $\mathcal{M}$ and Whittaker $\mathcal{W}$ functions (or equivalently suitable confluent hypergeometric functions or Kummer functions), multiplied by a power of $\xi$ and an exponential term with purely imaginary argument. The subsequent growth/decay of these oscillatory solutions can be described in the limit as $\xi \rightarrow \infty$ analytically. The limiting growth/decay exponent is given by

$$
\begin{aligned}
p_{\infty}= & \frac{1}{2[1-(\kappa+2) E]}\{(5+4 \kappa) E-(4+3 \kappa) \\
& \left. \pm \sqrt{\frac{(1+\kappa)(1-E)}{E}}[(2 \kappa+1) E+1]\right\},
\end{aligned}
$$

where $u \sim \xi^{p_{\infty}}$ as $\xi \rightarrow \infty$. The plus-or-minus sign corresponds to the two independent solutions of (8). Solutions to (1) with $p_{\infty}<0$ exist in the slope range

$$
\begin{aligned}
\frac{c}{1-2 E} & <\sin \theta \\
& <\frac{c[16 E(1-E)+(1-2 E) \sqrt{1-12 E(1-E)}-1]}{2 E[1-13 E(1-E)]} .
\end{aligned}
$$

We note that the lower limiting value $\sin \theta=c /(1-2 E)$ is the slope angle for which $U_{B}=\sqrt{g_{B}^{\prime} D_{B}}$ in (5). The significance of this value is that, for lower values of $\theta$, the shallow water gravity wave that propagates with speed $U_{B}-\sqrt{g_{B}^{\prime} D_{B}}$ can propagate upstream; the values of $p_{\infty}$ in this regime should not be interpreted as growth of linear perturbations in the $x>x_{s}$ region, and it is only the larger slope angle in (10) that concerns us here.

For the baseline parameters relevant to the Ross Sea, $E=0.072, c=0.003$, and $\theta=0.01$, we find that $p_{\infty}=$ -1.17 (decay). The neutrally stable cases can be found when $p_{\infty}=0$, as given by the upper limit of (10), which for these same baseline parameters predicts growth of downstream (linear) perturbations for $\theta>0.071$. Numerical results for the integration of (7) subject to initial conditions $\Delta(1)=1, u(1)=1$, and $\gamma(1)=1$ are shown in Fig. 1 


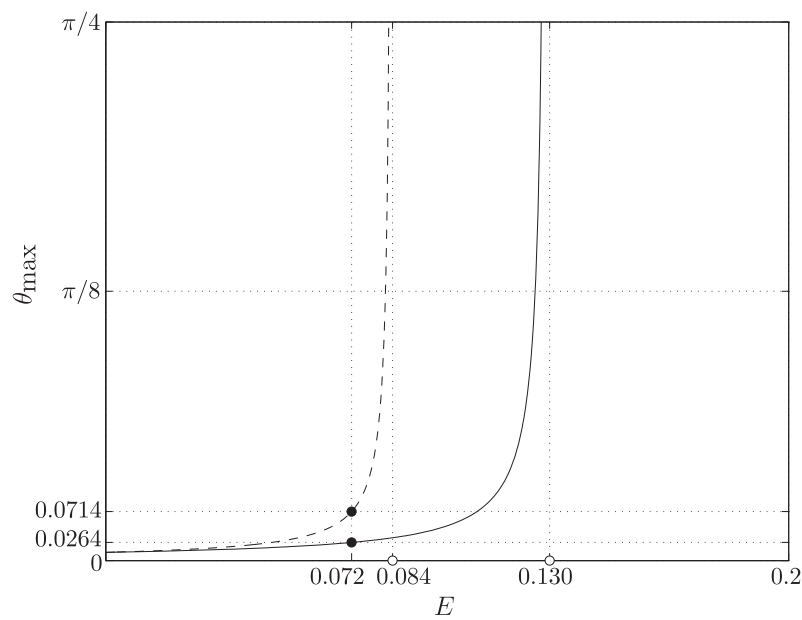

FIG. 2. The sensitivity of $\theta_{\max }$ to $E$. The original Holland (2011) model (1) is shown by the dashed line, and the present conservative model (2) is shown by the solid line. For practical values of $E$, the present conservative model exhibits low sensitivity to $E$. Both systems are approximately linear in their sensitivity to $c$. With the chosen base parameter $c=0.003$, the upper limits of (10) and (11) become greater than 1 for $E=0.083$ and 0.128 , respectively. The denominators vanish at $E=0.084$ and 0.130 , respectively, denoted by the white circles. For the chosen base parameter $E=0.072$, the predicted $\theta_{\max }=0.0714$ under the original Holland model and 0.0264 under the present conservative model (denoted by the black circles).

together with a comparison of the perturbation growth/ decay rates with the prediction in (9). Figure 1a also shows the evolving plume depth $D_{B}(1+\delta \Delta)$ for the base case values above and $\delta=A_{p}=0.25$ for later comparison of this linear theory with the nonlinear results of Fig. 3a (described in greater detail below). The long-time (linear) behavior is that the perturbations decay downstream, as demonstrated by Fig. 1b.

For the fully conservative form of the model equations [(1)], the relationship in (9) is modified somewhat with the upper limit for stability given by

$$
\sin \theta<\frac{4 c[6 E(2-E)+(1-E) \sqrt{1-3 E(2-E)}-1]}{E[8-33 E(2-E)]},
$$

and with our baseline parameters, this yields downstream growth for $\theta>0.0264$. The sensitivity of the maximum slope angle for stability $\theta_{\max }$ to $E$ is shown in Fig. 2. The sensitivity of $\theta_{\max }$ under the original Holland (2011) model (1), corresponding to the upper limit of (10), is shown as a dashed line. The sensitivity of $\theta_{\max }$ under the present conservative model (2), corresponding to the upper limit of (11), is shown as a solid line. The black circles denote the maximum stable slope angle for the base case $E=0.072$ and $c=0.003$ under the two models considered. We find $\theta_{\max }=0.0714$ and 0.0264
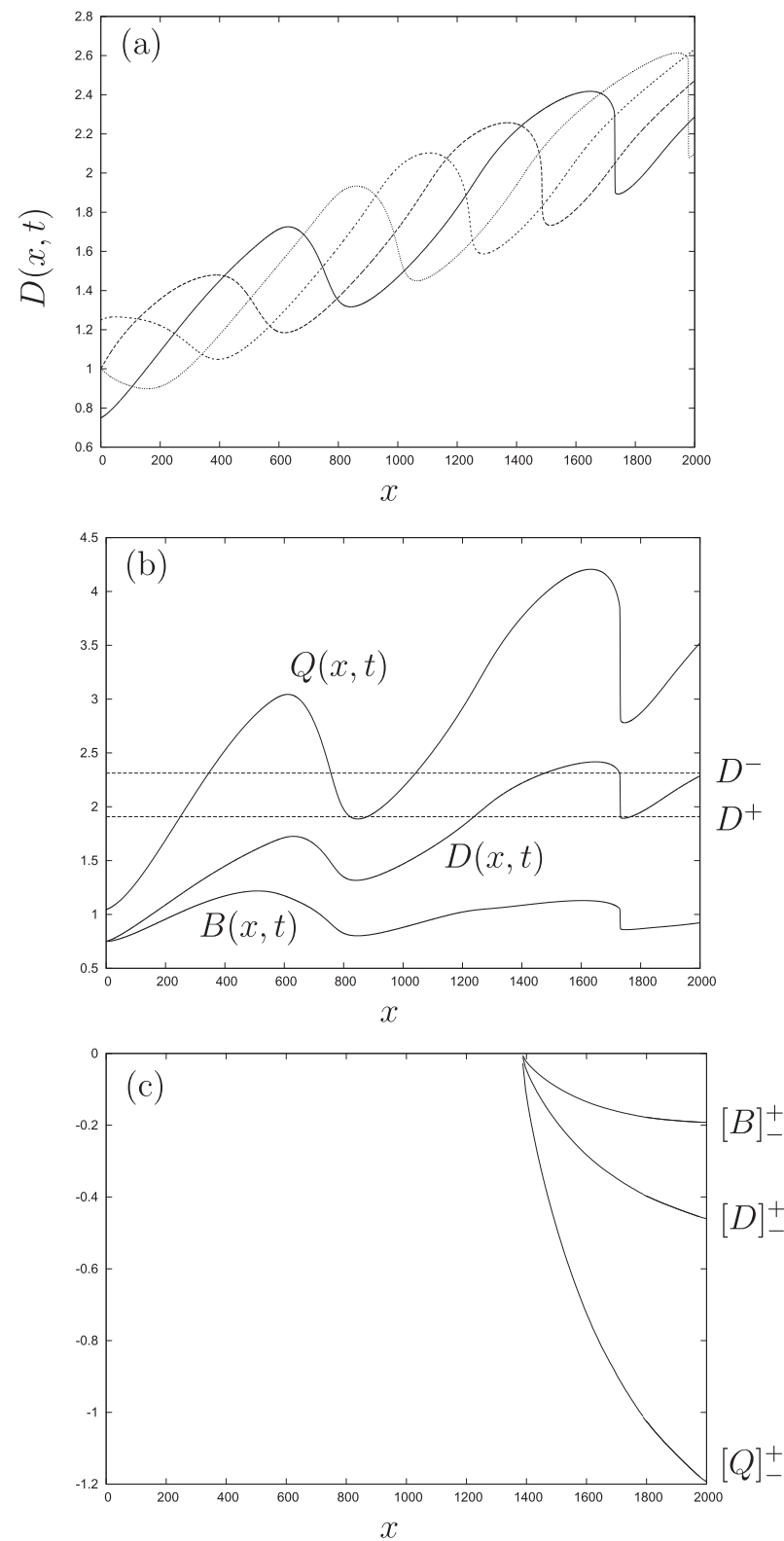

FIG. 3. (a) Evolution of the periodic (with unit period) $D(x, t)$ at $t=0,1 / 4,1 / 2,3 / 4$ with $A_{p}=0.25$ and $S=0.002588$; this nonlinear computation can be compared to the linear results of Fig. 1a. Line styles correspond to $t=0$ (dotted), $1 / 4$ (short dashed), 1/2 (long dashed), and $3 / 4$ (solid). Dimensional results are obtained by multiplying both axes by the initial plume depth of $100 \mathrm{~m}$. (b) The corresponding profiles for $Q(x, t)$ and $B(x, t)$ at $t=3 / 4$ with $A_{p}=$ 0.25 . Also shown are the predictions for the plume depth jump at the development of a shock in the profile. (c) The corresponding jumps in $Q, D$, and $B$ in the post-breaking region.

for the original and conservative models, respectively. The denominators of (10) and (11) vanish at $E=0.084$ and 0.130 , respectively (indicated by the white circles), but because $\sin \theta \leq 1$, in fact, (10) and (11) cease to predict real values for $\theta_{\max }$ at the slightly lower values 
$E=0.083$ and 0.128 , respectively, when $\theta_{\max } \approx \pi / 2$. We see that the present conservative model is considerably less sensitive to the entrainment coefficient than the original model for practical values of $E \approx 0.072$. As both (10) and (11) demonstrate that $\sin \left(\theta_{\max }\right)$ is linearly proportional to $c$, we see that both models have approximately linear sensitivity to $c$ because $\theta$ is small for practical applications.

The majority of ocean cascades occur on slopes of 0.001-0.01; of the 33 plumes considered by Ivanov et al. (2004), only two exceed the critical value above, so the oceanographic application of (1) is generally justified. In comparison, for the free axisymmetric plumes discussed by Scase and Hewitt (2012), linearized perturbations of frequency $\omega$ were shown to grow exponentially as $\exp \left(C \omega^{1 / 2} x^{2 / 3}\right)$, where $C$ is a constant. For such exponential growth, the numerical problem is ill posed and rapidly becomes swamped by high-frequency components. In the current case, even when perturbations grow downstream, they only do so algebraically, and the numerical initial-value problem remains well posed for all practical purposes.

\section{Numerical investigation of wave breaking}

Numerical solutions of (2) subject to (4) are obtained via a slope-limited monotone upstream-centered scheme for conservation laws (MUSCL), applying a finite-volume method to the integral form of the conservative equations. The scheme assumes a discontinuous solution across all element boundaries, but it does not explicitly solve the Riemann problem at each interface, applying instead the two-step method of Nessyahu and Tadmor (1990). Given that the model is stable and well posed, there is no difficulty in time marching the system from a $t=0$ state that consists of the steady solution, continuing until all transient behavior has decayed, to a periodic propagating wave state.

\section{a. Baseline simulation}

Figure 3a shows the downstream steepening of the plume depth during one period of the wave motion driven by source modulation. Over a full period, at each downstream location, we can define a maximum normalized gradient of the plume depth (relative to the steady state) $\sigma(x)=\max \left|D_{x}(x, t) / E \sin \theta\right|$, where this maximum is taken over an entire period. To avoid shock fitting, a straightforward way of defining the downstream location at which the wave has "broken" (i.e., where a shock develops) is to find the smallest value of $x=x_{b}$ such that $\sigma\left(x_{b}\right)=\mu$, where $\mu$ is a large value for the normalized gradient. However, to obtain robust results, one must ensure that the numerical resolution is increased in tandem with $\mu$ for this gradient to be accurately resolved by the numerical scheme. Typical values for the numerical results below are $\mu=50$ and an element size of 1.25 , and consistency is checked against $\mu=70$ and an element size of 0.625 .

In addition to the above gradient condition, one may also obtain the jump conditions across any such shock in the conservative model (2):

$$
\begin{aligned}
& S c_{s}[D]_{-}^{+}=[Q]_{-}^{+}, \quad S c_{s}[Q]_{-}^{+}=\left[\frac{Q^{2}}{D}+\frac{1}{2} B D\right]_{-}^{+}, \quad \text { and } \\
& S c_{s}[B]_{-}^{+}=\left[\frac{B Q}{D}\right]_{-}^{+},
\end{aligned}
$$

where $c_{s}$ is the shock propagation speed and the plus (minus) superscript indicates the state immediately ahead of (behind) the jump. For a given time during the periodic cycle, we may determine the $[\cdot]^{ \pm}$values numerically and therefore a comparison of the above three expressions for the shock speed $c_{s}$ allows an independent check of the numerical method. Alternatively, using the numerical estimates for $Q^{+}, Q^{-}, B^{+}$, and $B^{-}$, we may use (12) to derive the corresponding plume depths:

$$
\begin{aligned}
& D^{+}=\frac{B^{+} Q^{-}-B^{-} Q^{+}}{B^{-}-B^{+}}\left\{\frac{2 B^{+}}{B^{-}\left(B^{+}+B^{-}\right)}\right\}^{1 / 2} \text { and } \\
& D^{-}=B^{-} \frac{B^{+} Q^{-}-B^{-} Q^{+}}{B^{-}-B^{+}}\left\{\frac{2}{B^{+} B^{-}\left(B^{+}+B^{-}\right)}\right\}^{1 / 2}
\end{aligned}
$$

Figure $3 \mathrm{~b}$ shows this predicted jump in plume depth $D$ is consistent with the numerical solutions in the postbreaking region. Finally, in Fig. 3c, the downstream development of the jumps in $D, Q$, and $B$ are shown for the same parameter regime illustrated in Figs. $3 \mathrm{a}$ and $3 \mathrm{~b}$.

In Fig. 4a, we determine the dependence of the wavebreaking location $x_{b}$ as a function of the forcing amplitude $A_{p}$. For the baseline parameter values (shown as the dashed line), the location of wave breaking increases from approximately $50 \mathrm{~km}$ with $A_{p}=0.5$ (a $50 \%$ pulsation of plume variables at source) to $200 \mathrm{~km}$ with $A_{p}=$ 0.2 (a $20 \%$ pulsation of plume variables at source). We henceforth focus on the range $0.2 \leq A_{p} \leq 0.5$ because at lower $A_{p}$, the breaking occurs beyond any reasonable geophysical length scale, while at larger $A_{p}$, the breaking occurs ever closer to the plume origin, with no dependence upon model parameters other than $A_{p}$.

\section{b. Parameter sensitivity}

We now examine the impact of variations in $E, c, \theta$, $S$, and nonconstant slope angle on the wave-breaking 

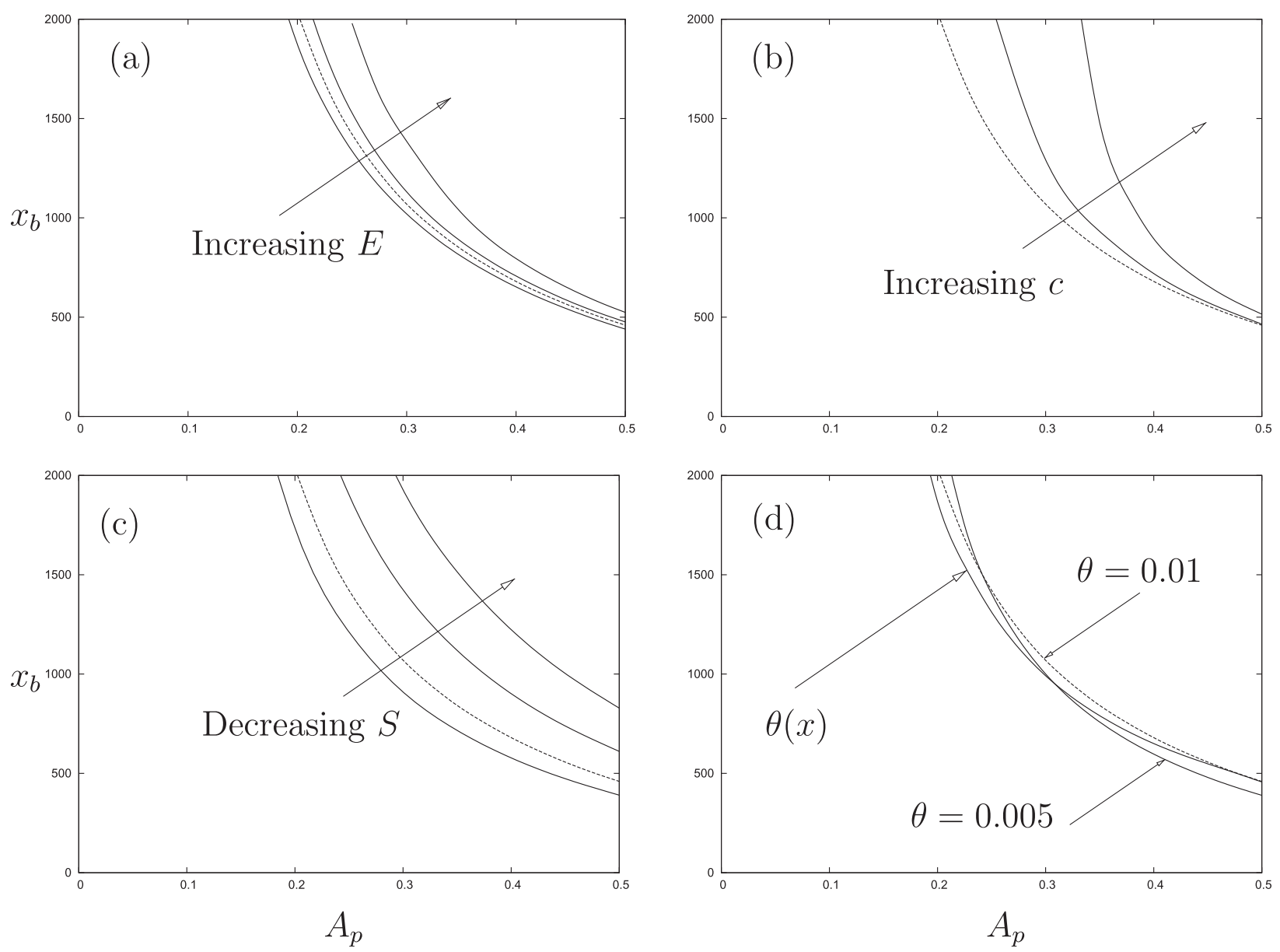

FIG. 4. Evolution of the numerically approximated point of wave breaking for increasing $A_{p}$ in the cases: (a) $E=0.05,0.072$ (dashed), 0.1 , $0.2 ; c=0.003 ; \theta=0.01 ; S=0.002588$. (b) $E=0.072 ; c=0.003$ (dashed), $0.005,0.007 ; \theta=0.01 ; S=0.002588$. (c) $E=0.072 ; c=0.003 ; \theta=0.01$; $S=0.003,0.002588$ (dashed), $0.002,0.0015$. (d) $E=0.072 ; c=0.003 ; \theta=0.01$ (dashed), $0.005 ; \theta(x) ; S=0.002588$, where $\theta(x)$ is given by $(14)$.

position, using the baseline results as the reference case and changing each of these variables in turn. The baseline results are shown as the dashed line in Figs. $4 \mathrm{a}-\mathrm{d}$. In addition, Fig. 4 shows the effect of variations in the entrainment coefficient $E=0.05,0.072$ (dashed), 0.1 , and 0.2 (Fig. 4a); the drag coefficient $c=0.003$ (dashed), 0.005 , and 0.007 (Fig. 4b); the parameter $S=0.003$, 0.002588 (dashed), 0.002, and 0.0015 (Fig. 4c); and the slope angle $\theta=0.01$ (dashed) and 0.005 (Fig. 4d). In Fig. 4d, we test the effect of a typical concave continental slope by testing the effect of a change in slope angle of the form:

$$
\begin{gathered}
\theta(x)=\theta_{0}-\frac{\theta_{0}-\theta_{1}}{2}\left[1+\tanh \left(\frac{x-500}{20}\right)\right], \\
\text { for } \theta_{0}=0.01 \text { and } \theta_{1}=0.005
\end{gathered}
$$

corresponding to a rapid transition from a slope angle of 0.01 at the inlet to an angle of 0.005 at a dimensional distance of approximately $50 \mathrm{~km}$ for an inlet plume depth of $100 \mathrm{~m}$. As discussed briefly in section 3, if the slope angle is reduced sufficiently [below a value of $2 c /(2-3 E) \approx 0.003$, for the baseline parameters], then one of the three waves in this hyperbolic model (2) propagates "upstream," corresponding to a shallow water gravity wave that can overcome the freestream speed of the plume. For slopes that eventually become this shallow, even in the absence of modulation of source conditions, a stationary shock solution is possible near the point where the local Froude number is unity. In the results of this paper, we instead concentrate on the nonstationary shocks (wave breaking) induced by modulation of the source conditions, which can occur for any slope gradient.

For all parameters, the sensitivity of the results is dependent upon $A_{p}$. At larger values of $A_{p}$, there is much less variation in the breaking position with the model parameters, because in this strongly driven regime, the breaking occurs closer to the plume origin, with $A_{p}$ being the dominant parameter. In this regime 
the wave breaking occurs on a length scale comparable to the distance $T U_{s}$ (approximately one wavelength), and over this (shorter) distance the parameters for entrainment, drag, and slope have a limited influence. Weakly driven waves break further downstream, and over these longer scales there is more variability with respect to the model parameters. Considering first the dependence on the entrainment coefficient $E$, the difference in the breaking position (relative to the baseline case of $E=0.072$ ) for $E=0.2$ ranges from $14 \%$ to $40 \%$ for $A_{p}$ from 0.5 to 0.25 (Fig. 4a). Changing $\theta$ between 0.01 and 0.005 causes a variation in breaking location of less than $15 \%$ across the range shown in Fig. $4 \mathrm{~d}$. This effect is clearly complex, with shallower slopes causing earlier breaking for high $A_{p}$ but later breaking for low $A_{p}$ (Fig. 4d).

The drag coefficient $c$ is highly important. Varying from the baseline value of $c$ from 0.003 to $c=0.007$, the wave breaking is (for practical purposes, given the resulting large length scales) completely removed from the system for $A_{p}<0.35$. For the three drag coefficients presented, the wave-breaking position can vary dramatically at even moderate pulsation amplitudes of $30 \%$, with no breaking found within a $200-\mathrm{km}$ domain for the higher drag coefficient of $c=0.007$. This is a simple result of the nonlinear drag parameterization in (1b), which discriminates against the faster-moving wave crests and thus delays breaking.

The parameter $S$ is also found to be influential. To a first approximation, the breaking position scales with $1 / S$, so halving $S$ will double the distance required for wave breaking. This parameter is always small in realistic applications; an upper bound can be found by considering semidiurnal tides $(T=12 \mathrm{~h})$ and thick plumes $\left(D=300 \mathrm{~m}, U=1 \mathrm{~m} \mathrm{~s}^{-1}\right)$, giving $S=0.007$. A measure of the advection time scale of the plume is given by $D_{s} / U_{s}$. Given a typical plume aspect ratio of 1000:1 (Fig. 3a), $1000 D_{s} / U_{s}$ is the time taken for a parcel of fluid to travel a typical plume length. The wavelength produced in the plume is the distance traveled by a parcel of fluid during one period of the source oscillation. Thus, as shown by Holland (2011), if $S \approx 0.001$, then $1000 D_{s} / U_{s} \approx T$ and the oscillating source will create a wave that is the length of the plume.

We now examine the influence that the choice of source pulsation has on the wave-breaking location. In the above results, we imposed (4) as a generic inflow boundary condition. We can of course consider unequal pulsation amplitudes in the form of

$$
\begin{aligned}
& B_{0}(t)=1+A_{p} \sin (2 \pi t) \quad \text { and } \\
& D_{0}(t)=1+\lambda A_{p} \sin (2 \pi t)
\end{aligned}
$$

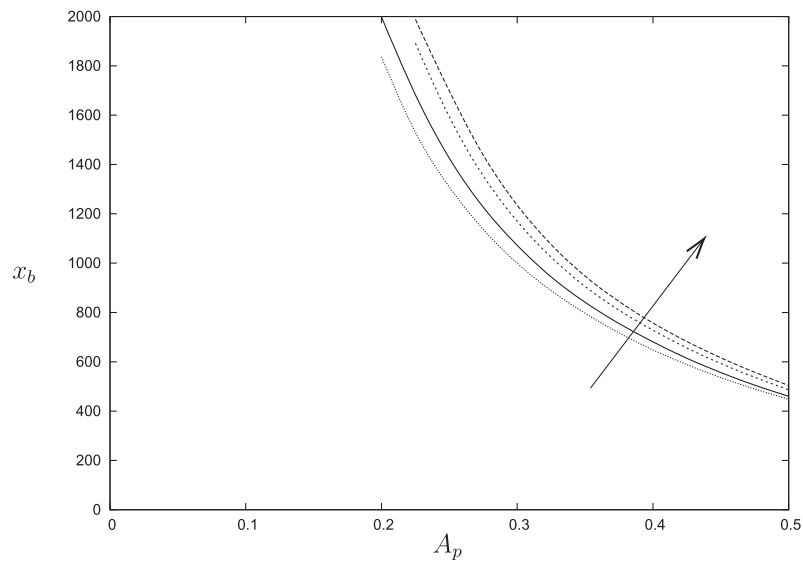

FIG. 5. Evolution of the point of wave breaking, for increasing $A_{p}$ and a range of unsteady source conditions [(15)] with $\lambda=1 / 4,1 / 2,3 / 2$, and 1 . The arrow indicates the direction of decreasing $\lambda$.

for a real constant $\lambda$, with $Q_{0}(t)$ determined as in (4). In Fig. 5, we compare the point of wave breaking for $\lambda=1 / 4,1 / 2,1,3 / 2$, where $\lambda=1$ corresponds to the (dashed line) results of Fig. 4a. This variation in the source conditions leads to differences of less than $20 \%$ in the predicted point of wave breaking; a variation that is comparable with that found for modified values of $E$ and $\theta$. This leaves $A_{p}, S$, and the drag coefficient $c$ as the dominant model parameters in terms of predicting the wave-breaking position.

\section{c. Diffusive effects}

Following Scase and Hewitt (2012), we can also consider the influence of diffusive effects on the evolution of the plume. Allowing for a velocity/buoyancy diffusion, with coefficients that scale with both plume depth and local velocity, introduces two associated dimensionless parameters $\epsilon$ and $\kappa$. The corresponding form of the governing system (2) becomes

$$
\begin{gathered}
S \frac{\partial D}{\partial t}+\frac{\partial Q}{\partial x}=E \sin \theta U \\
S \frac{\partial}{\partial t}(U D)+\frac{\partial}{\partial x}\left(U^{2} D+\frac{1}{2} B D\right) \\
=B \sin \theta-c U^{2}+\epsilon D \frac{\partial}{\partial x}\left(U D \frac{\partial U}{\partial x}\right), \quad \text { and } \\
S \frac{\partial B}{\partial t}+\frac{\partial}{\partial x}(U B)=\kappa \frac{\partial}{\partial x}\left(U D \frac{\partial B}{\partial x}\right) .
\end{gathered}
$$

The unsteady source conditions remain as stated in (4), and owing to the diffusive nature of the system, two further constraints are imposed downstream: 


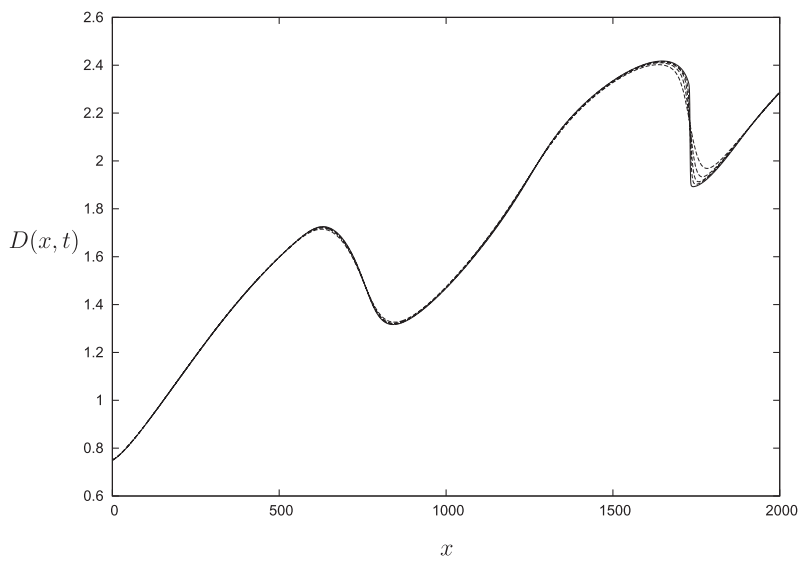

FIG. 6. A profile of the periodic (with unit period) dimensionless plume depth $D(x, t)$ at $t=3 / 4$ with $A_{p}=0.25$ and $S=0.002588$. The solid line is the diffusion-free result leading to a discontinuity in plume depth near $x \approx 1700$, while the diffusive cases $\epsilon=\kappa=1,1 / 2$, $1 / 4$, and $1 / 8$ are shown as the dashed lines. The smaller diffusion coefficients are associated with the steeper gradients.

$$
\frac{\partial U}{\partial x}=\frac{\partial B}{\partial x}=0 \quad \text { at } \quad x=x_{\infty}
$$

where the computational domain (indicated by the square brackets to be a closed range) is $x \in\left[0, x_{\infty}\right]$, for a suitably large value $x_{\infty}$. The previously noted steady solutions still solve this diffusive system exactly.

Choosing $\kappa=\epsilon=1$ explicitly scales the diffusion term to equal advection; increasing the diffusion far above this value would produce a fundamentally diffusive problem in which plume physics were unimportant, so we have investigated the range below this value. Figure 6 shows the influence of diffusion coefficients $\epsilon=\kappa=1,1 / 2$, $1 / 4$, and $1 / 8$. Diffusion does not affect the location of breaking, but it replaces the actual breaking with a diffusionlimited slope in all model variables at the same location. Even at these high diffusion coefficients, the effect of diffusion is localized and merely acts to mitigate the discontinuity in plume properties. As may be anticipated, the downstream steepening of the wave is ultimately limited by the diffusion coefficient, maintaining continuity of the solution. It is important to note that the removal of wave breaking by lateral diffusion does not detract from the oceanographic significance of the results. The peak in diffusion required to prevent wave breaking represents a vigorous local mixing that strongly resembles the expected effects of a breaking interfacial wave, and thus the basic result remains that strong mixing is expected at the wave-breaking location.

\section{Discussion}

The results are primarily affected by the nature of the imposed source oscillation, with wave breaking occurring closer to the source for higher-amplitude (higher $A_{p}$ ) or shorter-period (higher $S$ ) perturbations. In reality, the tidal forcing amplitude takes the full range from steady source to fully pulsed source for different plumes. Even in a single location, the plumes can be steady during neap tides and fully pulsed during spring tides. For example, in the Ross Sea, Antarctica, the spring tides advect the dense water source around such that it appears at the top of the slope for only $1 / 4$ of the tidal cycle, but during neap tides the dense water source is permanently present (Padman et al. 2009). The modeled position of wave breaking is relatively insensitive to entrainment, slope, and differential oscillation between the different source variables. Modeled wave breaking occurs closer to the source for lower seabed drag (lower $c$ ) and thicker or less-dense sources (higher $S$ ).

In our model, any positive diffusivity will prevent the development of shocks (which have a formally infinite gradient) in the plume variables, replacing them with a gradient in which wave-steepening processes are balanced by diffusion of momentum and buoyancy (Fig. 6). Thus, to the extent that our model equations represent the real world, diffusion will always prevent discontinuous plume properties, and wave breaking will instead be manifest as enhanced diffusion. Scaling $\kappa$ and $\epsilon$ by a characteristic velocity of $1 \mathrm{~m} \mathrm{~s}^{-1}$ and mixing length of $100 \mathrm{~m}$ produces dimensional diffusivities of $100 \mathrm{~m}^{2} \mathrm{~s}^{-1}$ in the upper limiting case tested here, $\kappa=\epsilon=1$. This high level of diffusivity is consistent with values derived from field observations of the ocean surface mixed layer on a horizontal length scale of $100 \mathrm{~km}$ (Thorpe 2005), so it is very much an upper bound for a study of plumes in the ocean interior (we are not aware of any observationbased estimates for lateral diffusion in dense ocean plumes). Choosing any value higher than this would explicitly scale the diffusive terms to be larger than advective terms, swamping the model results with diffusion. Any realistic value will be considerably smaller than this as the mixing length is the plume depth, not its length, and for these smaller values the results converge to (and are well approximated by) the nondiffusive case (Fig. 6), albeit without the development of a formal shock.

In the real oceans, breaking internal waves are a widely acknowledged source of diapycnal mixing. A complete suppression of internal wave breaking will depend upon the temporal and spatial variation of both the turbulence suppressing a nascent shock and the wavesteepening processes at play. With parameterized entrainment and lateral diffusion, our depth-integrated model is incapable of fully representing such effects. However, our model prediction of enhanced diffusion at the length scale of breaking internal waves suggests 
that this process could lead to enhanced local mixing in real-world plumes affected by a pulsed source.

Turbulence generally fluxes quantities down gradient. When diffusion is included, wave breaking is represented by the plume variables attaining the maximum permissible gradient for the chosen diffusivity. A pertinent question, then, is whether this gradient (and its resulting diffusion) is large relative to gradients (and thus diffusion) caused by other plume processes. The maximum gradients in our diffusive results are certainly found at the location of wave breaking (Fig. 6), so it is clear that wave breaking provides a much more vigorous downstream diffusion than is otherwise achieved in our scenario. This vigorous lateral diffusion is highly unusual for plumes. The break induces a downstream change in velocity of up to $50 \%$, which in the diffusive cases is spread over a length scale of $10 \mathrm{~km}$. In classical steady line plume theory, the velocity is spatially uniform, so it is difficult to conceive another situation, other than a hydraulic jump, in which such a strong downstream shear is found. It is possible that an external flow might impart such shear, but it is then debatable whether the resulting flow ought to be considered a buoyancy-controlled plume.

In dimensional terms, for an initial plume depth of $100 \mathrm{~m}$ and pulsation amplitude of $A_{p}=0.5$, the point of wave breaking occurs approximately $50 \mathrm{~km}$ from the source. The results of Holland (2011) did not demonstrate this same wave breaking; it was artificially damped by a numerical diffusion, which was uncontrolled in the sense that it was an emergent feature of the (arbitrary) computational mesh. Nevertheless, in this work we demonstrate that even with an explicit physically motivated (and controlled) downslope eddy diffusivity, the conclusions obtained from the diffusion-free equations still hold. A rapid downslope variation in plume properties is observed at the same wave-breaking location, but the downslope gradient of plume properties is inversely proportional to the eddy diffusion. At physically plausible values of the eddy diffusion, this downslope gradient remains significant.

\section{Conclusions}

Dense ocean plumes are an important source of deep and bottom water masses in the world's oceans, and these plumes are commonly affected by tides, which may cause a pulsing of the source of dense water. This study shows for the first time that such pulses lead to waves in the plume properties that can break at distances downstream that are geophysically relevant. To the extent to which this simple model is able to represent the real world, we expect such wave breaking to form an important, and previously unknown, source of mixing in dense ocean currents.

This study demonstrates that the unsteady line plume model is well posed, so that its implementation allows for a study of wave breaking. The results are primarily affected by the nature of the imposed source oscillation, with wave breaking occurring closer to the source for higher-amplitude or shorter-period perturbations. The modeled position of wave breaking is relatively insensitive to entrainment, slope, and differential oscillation between the different source variables. Modeled wave breaking occurs closer to the source for lower seabed drag and thicker or less-dense sources. The introduction of explicit diffusion into the model removes the breaking waves, replacing them with localized regions of intense horizontal mixing at the same location.

The results of this study suggest that wave breaking is an important process in dense water plumes. It is unclear to what extent the transmission and steepening of these gravity waves remains pertinent in a rotating system subject to baroclinic instability, where dense currents can break up into a series of eddies rather than maintaining a coherent plume (Cenedese et al. 2004). Because all ocean models feature both explicit and numerical diffusion, they cannot host actual wave breaking, though they may well represent the effect as intense horizontal mixing. This study raises the important prospect of a hitherto unconsidered process causing intense local mixing in dense ocean currents.

\section{REFERENCES}

Baines, P. G., and S. Condie, 1998: Observations and modelling of Antarctic downslope flows: A review. Ocean, Ice, and Atmosphere: Interactions at the Antarctic Continental Margin, S. S. Jacobs and R. F. Weiss, Eds., Antarctic Research Series, Vol. 75, Amer. Geophys. Union, 29-49.

Biton, E., J. Silverman, and H. Gildor, 2008: Observations and modeling of a pulsating density current. Geophys. Res. Lett., 35, L14603, doi:10.1029/2008GL034123.

Bo Pedersen, F., 1980: Dense bottom currents in rotating ocean. J. Hydraul. Div., 106, 1291-1308.

Cenedese, C., and C. Adduce, 2010: A new parameterization for entrainment in overflows. J. Phys. Oceanogr., 40, 1835-1850.

_ J. A. Whitehead, T. A. Ascarelli, and M. Ohiwa, 2004: A dense current flowing down a sloping bottom in a rotating fluid. J. Phys. Oceanogr., 34, 188-203.

Fer, I., U. Lemmin, and S. A. Thorpe, 2001: Cascading of water down the sloping sides of a deep lake in winter. Geophys. Res. Lett., 28, 2093-2096.

Hansen, B., and S. Østerhus, 2000: North Atlantic-Nordic Seas exchanges. Prog. Oceanogr., 45, 109-208.

Hewitt, R. E., and P. W. Duck, 2011: Pulsatile jets. J. Fluid Mech., 670, 240-259.

Holland, D. M., R. R. Rosales, D. Stefanica, and E. G. Tabak, 2002: Internal hydraulic jumps and mixing in two-layer flows. J. Fluid Mech., 470, 63-83, doi:10.1017/S002211200200188X. 
Holland, P. R., 2011: Oscillating dense plumes. J. Phys. Oceanogr., 41, 1465-1483.

Ivanov, V. V., G. I. Shapiro, J. M. Huthnance, D. L. Aleynik, and P. N. Golovin, 2004: Cascades of dense water around the world ocean. Prog. Oceanogr., 60, 47-98, doi:10.1016/ j.pocean.2003.12.002.

MacCready, P., W. E. Johns, C. G. Rooth, D. M. Fratantoni, and R. A. Watlington, 1999: Overflow into the deep Caribbean: Effects of plume variability. J. Geophys. Res., 104, $25913-$ 25935.

Morton, B. R., G. Taylor, and J. S. Turner, 1956: Turbulent gravitational convection from maintained and instantaneous sources. Proc. Roy. Soc. London, A234, 1-23, doi:10.1098/rspa.1956.0011.

Muench, R. D., L. Padman, A. Gordon, and A. Orsi, 2009: A dense water outflow from the Ross Sea, Antarctica: Mixing and the contribution of tides. J. Mar. Syst., 77,369-387, doi:10.1016/ j.jmarsys.2008.11.003.

Nash, J. D., H. Peters, S. M. Kelly, J. L. Pelegri, M. Emelianov, and M. Gasser, 2012: Turbulence and high-frequency variability in a deep gravity current outflow. Geophys. Res. Lett., 39, L18611, doi:10.1029/2012GL052899.

Nessyahu, H., and E. Tadmor, 1990: Non-oscillatory central differencing for hyperbolic conservation laws. J. Comput. Phys., 87, 408-463.
Orsi, A. H., G. C. Johnson, and J. L. Bullister, 1999: Circulation, mixing, and production of Antarctic Bottom Water. Prog. Oceanogr., 43, 55-109.

Padman, L., and S. Erofeeva, 2004: A barotropic inverse tidal model for the Arctic Ocean. Geophys. Res. Lett., 31, L02303, doi:10.1029/2003GL019003.

- , S. L. Howard, A. H. Orsi, and R. D. Muench, 2009: Tides of the northwestern Ross Sea and their impact on dense outflows of Antarctic Bottom Water. Deep-Sea Res. II, 56, 818-834, doi:10.1016/j.dsr2.2008.10.026.

Scase, M. M., and R. E. Hewitt, 2012: Unsteady turbulent plume models. J. Fluid Mech., 697, 455-480, doi:10.1017/jfm.2012.77.

— C. P. Caulfield, S. B. Dalziel, and J. C. R. Hunt, 2006: Timedependent plumes and jets with decreasing source strengths. J. Fluid Mech., 563, doi:10.1017/S0022112006001212.

Thorpe, S. A., 2005: The Turbulent Ocean. Cambridge University Press, 439 pp.

Wang, Q., S. Danilov, H. H. Hellmer, and J. Schröter, 2010: Overflow dynamics and bottom water formation in the western Ross Sea: Influence of tides. J. Geophys. Res., 115, C10054, doi:10.1029/2010JC006189.

Whitworth, T., and A. H. Orsi, 2006: Antarctic Bottom Water production and export by tides in the Ross Sea. Geophys. Res. Lett., 33, L12609, doi:10.1029/2006GL026357. 